Derechos reservados de El Colegio de Sonora, ISSN 1870-3925

\title{
Desarrollo forestal sustentable en Chihuahua, México: una estrategia multidimensional
}

\author{
Concepción Luján Álvarez* \\ Jesús Miguel Olivas García* \\ Hilda Guadalupe González Hernández* \\ Óscar Gómez Soto** \\ María de los Ángeles Cuautle Coyac ${ }^{* * *}$
}

Resumen: Establecer una estrategia multidimensional para lograr el desarrollo forestal sustentable en la región de clima templado frío en Chihuahua, México, fue el objetivo del presente estudio, que se obtuvo con la aplicación del modelo Multiconferencia de Búsqueda (MCB), basado en el sistema de organización por asociaciones de productores forestales (ejidatarios, comuneros y pequeños propietarios, incluidos indígenas y mestizos). La metodología participativa aplicada consideró el carácter social de la propiedad de los recursos forestales en México, y se apoyó en la colaboración activa de ejidos y comunidades y demás actores relacionados. La estrategia multidimensional establecida está integrada por las dimensiones ecológicas ambientales, socioculturales, económicas, político-institucionales y científico-tecnológicas, que incluye objetivos y acciones estratégicas que consideran las necesidades e intereses comunes de los involucrados en la búsqueda del desarrollo forestal regional, con visión de sustentabilidad.

\footnotetext{
* Profesores-investigadores de la Facultad de Ciencias Agrícolas y Forestales. Universidad Autónoma de Chihuahua. Km. 2.5 carretera Delicias-Rosales, C. P. 33000, Delicias, Chihuahua, México. Teléfono/Fax: (639) 472-27-26. Correos electrónicos: clujan@uach.mx/jolivas@uach.mx/gozzhhilda@mexico.com

** Profesional forestal. Secretaría de Medio Ambiente y Recursos Naturales. Delegación Chihuahua. Ave. de Las Américas \# 300-B, colonia San Felipe, C. P. 31240, Chihuahua, Chihuahua, México. Correo electrónico: goso661120@chihuahua.com

*** Ingeniero forestal. Servicios Técnicos Forestales. Correo electrónico: al50435@uach.mx
} 
Palabras clave: desarrollo sustentable, desarrollo forestal, sustentabilidad, comunidades forestales sustentables, planeación estratégica participativa, desarrollo forestal regional.

Abstract: The implementation of a multidimensional strategy for sustainable forestry development in the cold temperate weather region in Chihuahua, Mexico was the objective of this study, through the application of the Multi-Conference Search Model, based on the organization system of forestry producer associations (ejidatarios, comuneros, and small landowners, including indigenous and mestizo people). The participative methodology applied took into account the public nature of forest resource ownership in Mexico. Ejidos, communities, and other relevant actors were actively involved in this process. The multidimensional strategy designed incorporates the ecological, environmental, sociocultural, economic, political, institutional, scientific and technological dimensions, which include objectives and strategic actions that consider needs and common interests of the entities involved in the pursuit of sustainable regional forestry development.

Key words: sustainable development, forestry development, sustainability, sustainable forestry communities, strategic participative planning, regional forestry development.

\section{Introducción}

Las políticas ambientales internacionales muestran una tendencia clara a adoptar modelos de desarrollo acordes con los principios de sustentabilidad, y a buscar acuerdos de cooperación para solucionar la problemática del medio ambiente (Vanhanen et al. 2005). En México, anteriormente la estrategia para aprovechar la riqueza forestal estuvo basada en la concesión para la explotación de los bosques a compañías privadas, con participación escasa de los dueños en el proceso productivo, con la limitación consecuente de los beneficios económicos para ellos. En contraste, en la actualidad el sector forestal se expresa en el Plan Nacional de Desarrollo y el Programa Nacional de Medio Ambiente y Recursos Naturales 2001-2006, y se sustenta en una percepción de largo plazo, que incluye e integra a todos los actores del ramo 
en torno a un objetivo central: el desarrollo forestal sustentable. En congruencia con ello, el gobierno federal definió a los bosques y al agua como asuntos de seguridad nacional (Secretaría de Medio Ambiente y Recursos Naturales, SEMARNAT-Comisión Nacional Forestal, CONAFOR 2001).

En relación con esta estrategia nacional y regional, los ejidos y comunidades forestales representan un componente importante en la cadena productiva del sector y, a la vez, constituyen un sistema sociocultural y ecológico clave para su desarrollo con una visión de sustentabilidad; sin embargo, no han logrado obtener los beneficios esperados con dicho enfoque (Bray et al. 2003). Asimismo, la gente local no ha tenido oportunidad de participar activamente en los procesos de toma de decisiones relacionadas con el diseño, implementación y evaluación de planes y programas para el desarrollo forestal sustentable (Luján et al. 2006).

Particularmente en Chihuahua, en relación con el contexto sociopolítico, se percibe que a través de los años el control de la producción en los ejidos y comunidades forestales ha estado en manos de un número reducido de personas, quienes se benefician de la comercialización de los productos, y los ejidatarios reciben poca o nula compensación económica por el uso de sus recursos. Asimismo, es de resaltar que la sierra Tarahumara, área del estudio, posee una gran riqueza cultural y biológica; sin embargo, también presenta problemas sociales y políticos que giran en torno a la actividad forestal (Guerrero et al. 2000a; 2000b). En relación con la población que la habita, el grupo indígena predominante es el tarahumara o rarámuri, aunque también existen tepehuanes, guarijíos y pimas, que viven dispersos en rancherías dentro de los ejidos, y así coexisten con los mestizos, quienes en cambio se concentran en áreas urbanas y en núcleos de población de los ejidos (Guerrero et al. 2000b).

Por otra parte, la vida cultural de los indígenas de Chihuahua tiene una relación estrecha con sus recursos naturales, y es evidente que su visión del manejo de ellos, basada en el respeto e interés común, no coincide con la de los mestizos, quienes buscan satisfacer sus intereses económicos en el corto plazo y a la vez despliegan mayor dinamismo en las actividades económicas, provocando así algunos conflictos entre ambos grupos (Guerrero et al. 2000a).

En Chihuahua predomina el régimen de propiedad comunal (ejidos y comunidades forestales), que representa aproximadamente 80 por ciento de la superficie estatal comprendida en más de siete millones de hectáreas de bosque de clima templado frío (Secretaría de Agricultura y Recursos Hidráulicos, SARH 1994). Ante estas condiciones, es necesario diseñar e implantar estrategias estatales y regionales con visión multidimensional y de sustenta- 
bilidad para el sector forestal. Lo anterior, con el propósito de favorecer la coordinación de esfuerzos y recursos para alcanzar objetivos regionales, ya que los procesos de planeación en este ámbito están fundamentalmente orientados a la atención de problemas y necesidades locales, y con el apoyo de los actores relacionados con el desarrollo forestal se podrá favorecer la integración de su cadena productiva.

Por lo tanto, el desarrollo forestal sustentable debe de avanzar con un enfoque holístico, considerando como eje el impulso de la forestería comunitaria en México, ya que ésta representa aproximadamente 80 por ciento de la superficie nacional (Bray et al. 2003), y los ejidos y comunidades viven con las consecuencias inmediatas de cualquiera de sus acciones o decisiones. Por ello es necesaria una visión múltiple, sistémica y de largo plazo. Por tanto, la metodología de la MCB se convierte en una opción fundamental, para la planeación estratégica participativa, que toma en cuenta los elementos citados y puede brindar bases y resultados para lograr una visión prospectiva y avanzar hacia futuros deseables y alcanzables, en relación con el desarrollo forestal sustentable en México (Luján et al. 2006). En función de lo anterior, es importante promover el cambio de paradigma consistente en maximizar rendimientos, para dar paso a la búsqueda de una estrategia multidimensional que facilite el equilibrio y optimización de la productividad del ecosistema forestal con equidad social y cultural, viabilidad económica, protección y conservación de los recursos naturales y el ambiente, contribución científica y tecnológica, y así lograr una instrumentación efectiva de la política forestal que impulse su desarrollo regional con criterios de sustentabilidad (Consejo Técnico Consultivo Forestal de Chihuahua, СтсFCH 1996).

El objetivo de este estudio fue establecer una estrategia multidimensional que integre las dimensiones ecológico-ambiental, sociocultural, económica, político-institucional y científico-tecnológica, para lograr el desarrollo forestal sustentable de Chihuahua. Ésta incluye necesidades, deseos e intereses de los diferentes actores del sector y, a la vez, orienta las diversas acciones estratégicas para avanzar hacia tal fin. Asimismo, se espera que apoye a los responsables regionales para alcanzarlo.

\section{Metodología}

Para definir e implantar la estrategia multidimensional fue necesario apoyarse en principios del desarrollo participativo y en metodologías que permitan asegurar una colaboración democrática, con visión holística en los procesos, para el diseño de este instrumento de política forestal. 
Ubicación geográfica del área de estudio

Está localizada en la región forestal de bosque de clima templado frío de Chihuahua, en la provincia fisiográfica Sierra Madre Occidental y principalmente en las subprovincias Gran Meseta y Cañones Chihuahuenses, Sierras y Cañadas del Norte y Sierras y Llanuras Tarahumaras, entre los $25^{\circ} 35^{\prime}$ y $30^{\circ} 30^{\prime}$ de latitud norte y los $106^{\circ} 30^{\prime}$ y $109^{\circ} 07^{\prime}$ de longitud oeste (véase figura 1).

\section{Definición de unidades de estudio}

Se definieron con base en el sistema de organización forestal, integrado por asociaciones regionales de productores, establecido por el Fideicomiso Chihuahua Forestal (véase figura 1).

Al considerar las necesidades del estudio y las condiciones geográficas, socioeconómicas y la naturaleza del modelo participativo $\mathrm{MCB}$, se definieron tres zonas de trabajo: norte, centro y sur. Para cada una se realizaron dos conferencias de búsqueda (СB) (Emery y Ronald 1996), que incluyeron a asociaciones de productores forestales, integradas por ejidatarios, comuneros y pequeños propietarios; se agruparon según criterios de similitud de las dimensiones socioculturales, económicas y ecológicas, de manera que fueron seis conferencias en total o talleres participativos, que representaron toda el área de estudio con una participación promedio de 25 personas en cada una.

\section{Actores principales}

En la fase inicial del proceso de planeación estratégica participativa, para el establecimiento de la estrategia multidimensional, los actores fueron los dueños de los recursos forestales (ejidos y comunidades), ya que ellos representan el eslabón base de la cadena productiva del sector, con su participación se logró uno de los principios básicos del desarrollo sustentable. Además, así se asegura la democracia, como un proceso de toma de decisiones de “abajo hacia arriba”. Después, se involucró a otros actores e instancias representadas en el Consejo Estatal Forestal del Estado de Chihuahua (CEFECH), como industriales (empresas privadas), dependencias estatales y federales, organizaciones no gubernamentales, prestadores de servicios técnicos forestales y el sector educativo y de investigación, entre otros. 


\section{Figura 1}

Ubicación geográfica del área de estudio, mapa regionalizado

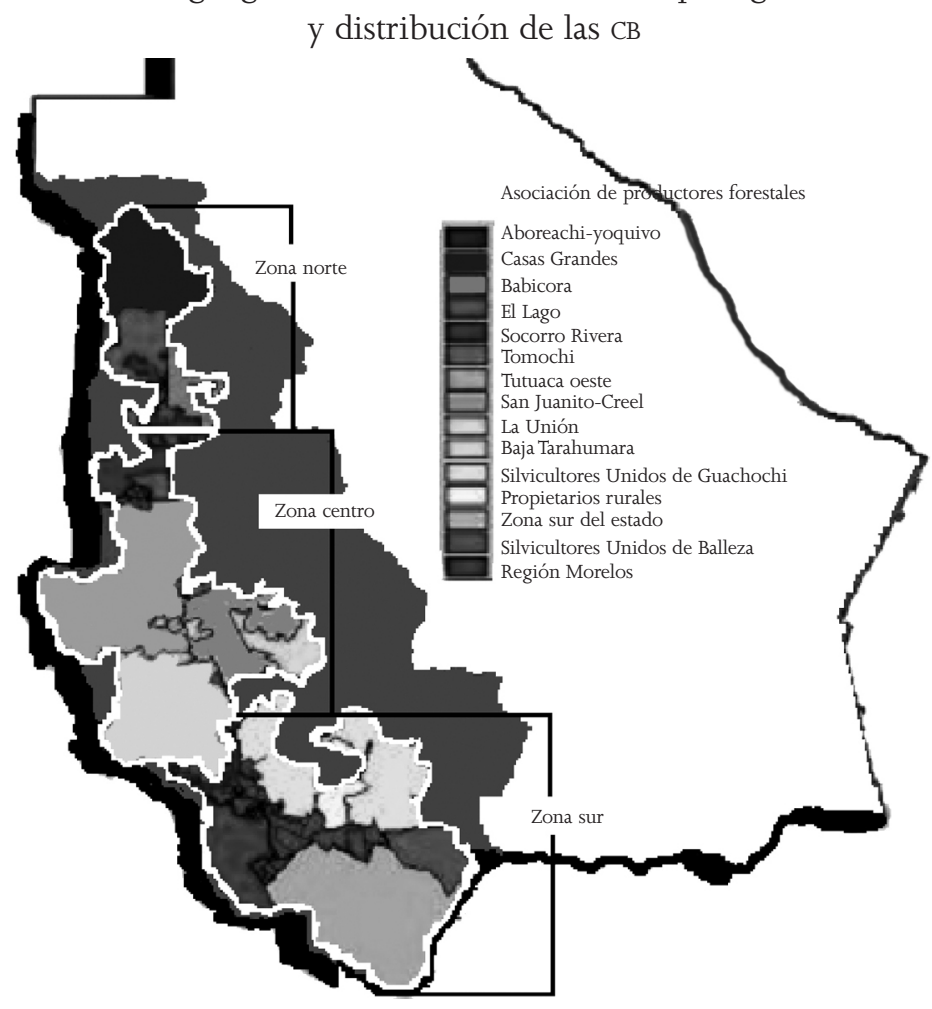

Unidades de estudio y distribución de las conferencias de búsqueda

\begin{tabular}{|c|c|c|}
\hline Zona & Asociación de productores forestales & Conferencia de búsqueda \\
\hline \multirow[t]{2}{*}{ Norte } & Casas Grandes & $\begin{array}{l}\text { Conferencia } 5 \\
\text { Nuevo Casas Grandes }\end{array}$ \\
\hline & $\begin{array}{l}\text { El Largo } \\
\text { Socorro Rivera } \\
\text { Alta Babicora }\end{array}$ & $\begin{array}{l}\text { Conferencia } 6 \\
\text { Madera }\end{array}$ \\
\hline \multirow[t]{2}{*}{ Centro } & $\begin{array}{l}\text { San Juanito-Creel } \\
\text { La Unión } \\
\text { Baja Tarahumara } \\
\text { Aboreachi-Yoquivo }\end{array}$ & $\begin{array}{l}\text { Conferencia } 1 \\
\text { Bocoyna }\end{array}$ \\
\hline & $\begin{array}{l}\text { Tutuaca oeste } \\
\text { Tomochi }\end{array}$ & $\begin{array}{l}\text { Conferencia } 2 \\
\text { Basaseachi }\end{array}$ \\
\hline \multirow[t]{2}{*}{ Sur } & $\begin{array}{l}\text { Productores y responsables técnicos de la zona sur del estado } \\
\text { Silvicultores Unidos de Balleza }\end{array}$ & $\begin{array}{l}\text { Conferencia } 3 \\
\text { Parral }\end{array}$ \\
\hline & $\begin{array}{l}\text { Silvicultores Unidos de Guachochi } \\
\text { Propietarios rurales } \\
\text { Región Morelos }\end{array}$ & $\begin{array}{l}\text { Conferencia } 4 \\
\text { Guachochi }\end{array}$ \\
\hline
\end{tabular}

Fuente: Fideicomiso Chihuahua Forestal (2003). 


\section{Diseño y aplicación de la multiconferencia de búsqueda}

La filosofía que sustenta el diseño de la estrategia multidimensional se basó en los principios siguientes: pensamiento estratégico, visión holística y democracia participativa. Para ser congruentes con dichos principios, se consideró la aplicación de la $\mathrm{MCB}$, integrada por un conjunto de $\mathrm{CB}$, que constituye una metodología participativa, flexible y versátil (Luján 2001). En el presente estudio, la МСB se fundamentó en la aplicación de seis Св (véase figura 2), cuyo proceso de aplicación se describe a continuación.

\section{Figura 2}

Estructura de aplicación de la MCB y obtención de la estrategia multidimensional para el desarrollo forestal sustentable

Multiconferencia de búsqueda

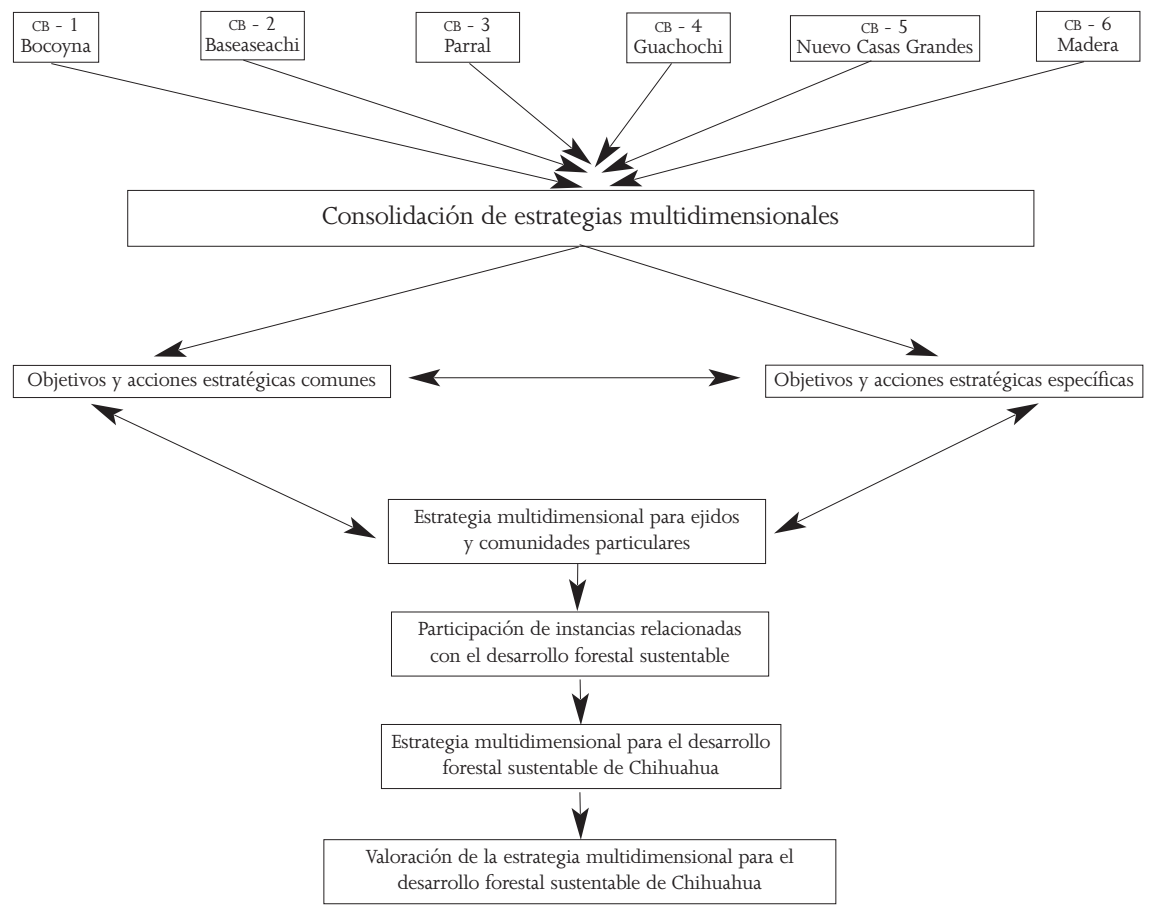

Fuente: Luján (2001). 
Descripción de las fases de la metodología de CB y su aplicación

Incluye las fases descritas a continuación (Luján et al. 2000):

- Acceso y comunicación con el sistema de referencia. Esto es un paso inicial para la CB, es con apoyo de personas clave, por lo cual se estableció contacto con ellas en las zonas forestales mencionadas, para facilitar la organización y aplicación del proceso participativo.

- Elección de los participantes. Ejidatarios, comuneros y pequeños propietarios fueron seleccionados con la ayuda y referencia de informantes clave, como prestadores de servicios técnicos forestales y coordinadores de asociaciones regionales, así como por habitantes o autoridades de la misma comunidad o ejido. La selección se basó en los criterios siguientes: miembro del ejido o comunidad, interés por el desarrollo de su comunidad, liderazgo, disponibilidad para participar, conocimiento del área y experiencia en la actividad forestal, entre otros. Así los participantes podrían ser miembros del ejido o comunidad, sin ser necesariamente autoridades locales.

- Diseño de la св. Se hizo describiendo el propósito, actividades de los participantes (generación de datos, labores del diagnóstico y acciones de planeación) y tiempo disponible.

- Aplicación de la CB. El proceso consistió en las etapas siguientes (véase figura 3):

Figura 3

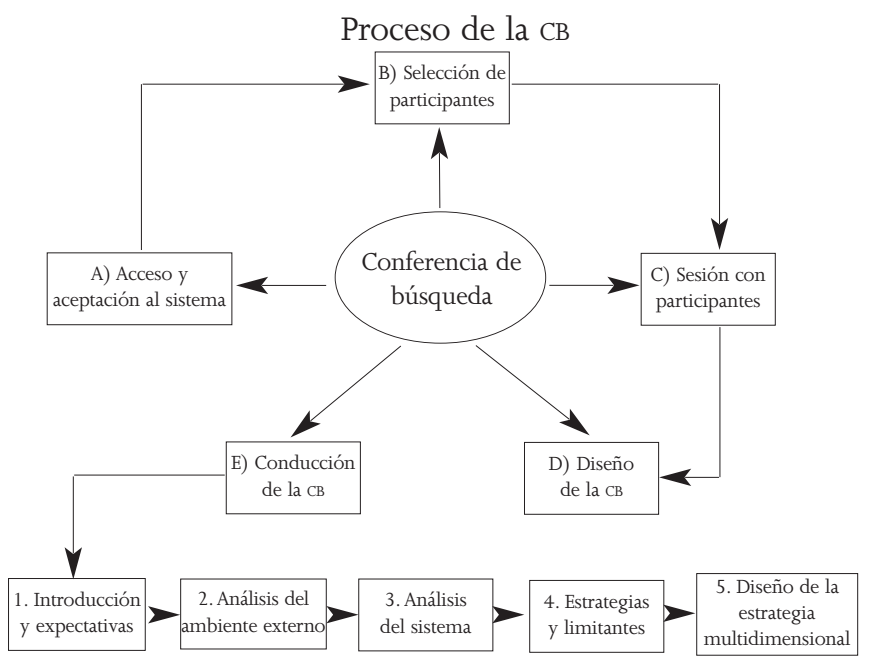

Fuente: Luján (2001). 
- Introducción y sesión de información: los administradores del proceso presentaron una visión de los objetivos, estructura, proceso, itinerario y detalles esenciales de la reunión.

- Análisis del ambiente externo: los participantes analizaron su ambiente externo. La pregunta fue: ¿qué cambios han ocurrido en el entorno global en los últimos cinco a siete años, que usted considera significativos? Además, se definieron escenarios de futuros más probables y deseables, así como oportunidades y amenazas.

- Análisis del ambiente interno o sistema: los participantes analizaron su ambiente interno en la comunidad o ejido, con el propósito de examinar su historia e identificar su carácter distintivo y competencias. La pregunta fue: ¿qué eventos mayores o desarrollos históricos son significativos para que la comunidad sea lo que es hoy? Inmediatamente después de la sesión de la historia se analizaron los aspectos comunitarios que se desea mantener, eliminar y crear. Además, se definieron escenarios de futuros más probables y deseables, así como fortalezas y debilidades.

- Integración del sistema y el ambiente externo: limitantes. En esta fase, ellos identificaron los obstáculos mayores para alcanzar su futuro deseable. Después, se generaron estrategias que permitan convertir a las limitantes en acciones positivas para lograr las metas.

- Evaluación de estrategias: una vez que en plenaria se reportaron y revisaron las estrategias para sobrepasar las barreras, se les preguntó: ¿es realizable el futuro más deseable de la comunidad? Una vez que ellos determinaron que sí, sus declaraciones se convirtieron en objetivos estratégicos, que fueron la base para elaborar propuestas para su implementación y financiamiento.

- Priorización de objetivos estratégicos: con base en las necesidades e intereses de los actores involucrados, ellos mismos establecieron una priorización de los objetivos estratégicos, sustentada en los criterios siguientes: a) enfocar una necesidad inmediata y crucial del sistema; b) involucrar a muchos residentes y actores del sistema de referencia; c) crear una visibilidad e imagen para atraer recursos nuevos y apoyo adicional; d) alcanzar los objetivos con los recursos disponibles o adicionales limitados y e) crear un ambiente favorable y facilitar el alcance de otros objetivos.

- Diseño de la estrategia multidimensional: esta tarea conjuntó el trabajo previo, integrando el ambiente externo y el sistema.

Para realizar esta fase, cada participante seleccionó el objetivo en el que deseaba trabajar, según su conocimiento y experiencia, y por lo tanto, en el que estaría comprometido (véase figura 4). 


\section{Figura 4}

Sesión de una conferencia de búsqueda

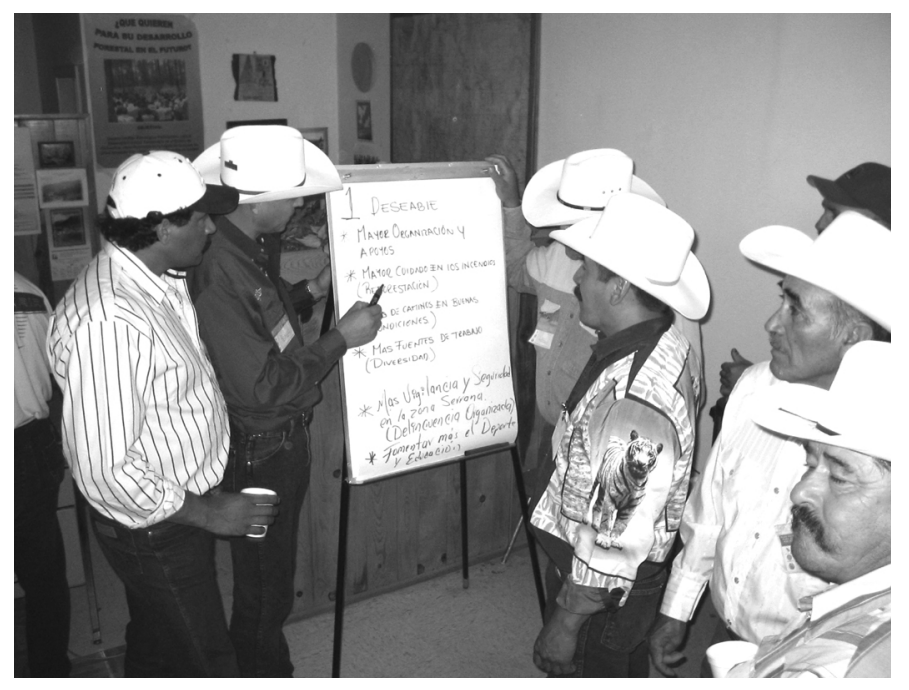

Los elementos que integran la estrategia multidimensional son los objetivos y acciones estratégicas, los responsables, tiempo de realización, lugar, necesidades de capacitación, recursos y apoyos requeridos y evaluación de avances y resultados.

Integración de la estrategia multidimensional

Una vez obtenida la estrategia multidimensional, que incluye intereses y necesidades comunes en todo el sistema o región en Chihuahua y los requerimientos específicos para cada zona forestal, se procedió a su consolidación, lo cual consistió en identificar objetivos compartidos por las unidades de estudio.

Posteriormente, se convocó a un taller forestal para presentar y retroalimentar la estrategia multidimensional consolidada por los ejidatarios, comuneros y pequeños propietarios, y generar objetivos estratégicos y acciones relacionadas con todos los actores de la cadena productiva. Después se convocó a tres productores forestales de cada una de las unidades de trabajo donde se realizaron las $\mathrm{CB}$, así como al menos a un representante de cada instancia relacionada con el sector en el estado. El resultado fue la integración de ideales de todos los participantes. Además, se definieron las accio- 
nes para continuar con la implantación, temporalidad y colaboración de instituciones relacionadas, sugeridas por los actores del proceso. Lo anterior, con la finalidad de establecer puntualmente los aspectos esenciales para poner en marcha la estrategia multidimensional, así como un mecanismo de seguimiento para el monitoreo y evaluación de lo planeado. Esto permitirá asegurar un proceso dinámico, permanente y holístico, a través del modelo activo-adaptativo entre los ambientes externo e interno.

\section{Validación de la estrategia multidimensional}

Una vez consolidada la estrategia multidimensional, se convocó a un segundo taller cuyo propósito fue validarla en Chihuahua, para lograr el desarrollo forestal sustentable. El resultado fue su validación y retroalimentación definitiva, así como de las acciones futuras para su instauración.

\section{Resultados y discusión \\ Escenarios probables y deseables}

Los actores del proceso de planeación, fundamentalmente ejidatarios, comuneros y pequeños propietarios en el análisis del ambiente externo e interno, diseñaron un escenario del futuro más probable, considerando los aspectos socioeconómicos, biológicos y culturales que interactúan en el contexto externo y que de alguna forma intervienen o influyen en el sistema. Las declaraciones que integran el escenario más probable, proyectado para la región de bosque de clima templado frío de Chihuahua fueron: a) deterioro, contaminación y disminución de los recursos naturales; b) aumento de la emigración por falta de fuentes de empleo en la región; c) incremento del narcotráfico; d) mayor tala clandestina y e) baja rentabilidad en el aprovechamiento de los recursos forestales.

Por otra parte, el proceso permitió que los actores buscaran ideales de acuerdo a sus deseos e intereses, con el propósito de establecer direcciones y estrategias individuales y colectivas nuevas en la búsqueda compartida de un futuro más deseable y modificable. Como resultado de la consolidación del escenario descrito, se aprecia el deseo por transitar hacia un desarrollo forestal sustentable, cambiar las dimensiones ecológicas, económicas y sociales para satisfacer los requerimientos, deseos e intereses de los actores involucrados. También resalta su necesidad y anhelo de hacer del desarrollo forestal una alternativa importante para lograr el bienestar cuidando los bosques y a la vez ser más competitivos, tanto a escala nacional como internacional. 


\section{Oportunidades, amenazas, fortalezas y debilidades}

Los actores analizaron el contexto externo e interno actual, esto es, revisaron las oportunidades y amenazas del ambiente externo, presentadas en el cuadro 1, y las fortalezas y debilidades principales de su región, incluidas en el cuadro 2.

\section{Cuadro 1}

Oportunidades y amenazas: ambiente externo

\begin{tabular}{|l|l|}
\hline \multicolumn{2}{|c|}{ Ambiente externo } \\
\hline Oportunidades & Amenazas \\
\hline $\begin{array}{l}\text { EL Tratado de Libre Comercio de América } \\
\text { del Norte (TLCAN) y el tipo de cambio } \\
\text { favorecen la importación de madera } \\
\text { a industriales locales y nacionales a } \\
\text { precios más baratos }\end{array}$ & $\begin{array}{l}\text { EL TLCAN y el tipo de cambio favorecen } \\
\text { la importación de una gran cantidad } \\
\text { de productos maderables a menor costo } \\
\text { que los nacionales, lo que los pone } \\
\text { en desventaja }\end{array}$ \\
\hline $\begin{array}{l}\text { Programas de apoyo del gobierno } \\
\text { federal y estatal }\end{array}$ & $\begin{array}{l}\text { Deficiencias en la aplicación } \\
\text { de la normatividad forestal }\end{array}$ \\
\hline $\begin{array}{l}\text { Programas del gobierno federal en la } \\
\text { capacitación a productores forestales }\end{array}$ & $\begin{array}{l}\text { Los costos, excesiva normatividad y trámites } \\
\text { para obtener la certificación forestal }\end{array}$ \\
\hline $\begin{array}{l}\text { La Ley General de Desarrollo Forestal } \\
\text { Sustentable (LGDF) }\end{array}$ & Falta de información a productores \\
\hline $\begin{array}{l}\text { La certificación forestal favorece } \\
\text { el desarrollo, por la incursión } \\
\text { a mercados internacionales }\end{array}$ & $\begin{array}{l}\text { Programas de apoyo inoportunos } \\
\text { para el sector forestal }\end{array}$ \\
\hline Generación y avances en la tecnología & La corrupción \\
\hline Funte: Iuín (2001)
\end{tabular}
Fuente: Luján (2001).

Para avanzar en el desarrollo forestal sustentable es importante minimizar las amenazas identificadas por ejidatarios, comuneros y pequeños propietarios: a) apertura económica a la importación de productos a precios más bajos; b) deficiencia en la aplicación de la normatividad forestal; c) costos, normatividad y gestiones excesivas para la certificación forestal; d) programas ineficientes y e) exceso de trámites con procesos burocráticos en las dependencias federales y estatales.

Por otro lado, es necesario fortalecer los aspectos y factores identificados como debilidades del sistema: a) deficiencias en el manejo de los recursos forestales; b) aumento de los costos de producción; c) escasa infraestructura caminera; d) bajo volumen aprovechado; e) ausencia de fuentes de empleo y f) falta de participación de ejidatarios, comuneros y pequeños propietarios. 


\section{Cuadro 2}

Fortalezas y debilidades del sector forestal en el estado

\begin{tabular}{|l|l|}
\hline \multicolumn{2}{|c|}{ Análisis del ambiente del sector forestal (sistema) } \\
\hline Fortalezas & Debilidades \\
\hline Cantidad de recursos forestales y madera de calidad & Deficiencias en el manejo de los recursos forestales \\
\hline $\begin{array}{l}\text { El recurso humano y su experiencia en el } \\
\text { aprovechamiento de los bosques }\end{array}$ & Las malas condiciones de la infraestructura caminera \\
\hline La infraestructura caminera & Tala clandestina \\
\hline La operación de asociaciones de productores forestales & El narcotráfico \\
\hline Belleza escénica y atractivos turísticos & $\begin{array}{l}\text { Falta de interés colectivo y por tanto de unión entre } \\
\text { productores }\end{array}$ \\
\hline $\begin{array}{l}\text { La necesidad e interés de la gente para buscar más } \\
\text { opciones de desarrollo }\end{array}$ & Carencia de fuentes de empleo \\
\hline $\begin{array}{l}\text { Apoyos económicos de programas gubernamentales } \\
\text { federales y estatales }\end{array}$ & Altos costos de producción \\
\hline
\end{tabular}

Por lo tanto, las principales acciones identificadas, de acuerdo con el análisis del contexto actual, son las siguientes: a) aprovechar la cantidad de madera de calidad, para crear empresas más eficientes, capaces de participar en nichos específicos de mercado con productos competitivos y crear fuentes de empleo; b) emplear el capital humano calificado y su experiencia en el aprovechamiento de los recursos e implantar acciones para buscar la certificación forestal y así competir en los mercados internacionales; c) utilizar y aplicar adecuadamente los subsidios o apoyos directos, tales como los administrados por la CONAFOR. Todo para coadyuvar al financiamiento de las diversas actividades del sector forestal, que permitan desarrollar, favorecer e impulsar su producción, conservación y restauración, así como elevar su rentabilidad; d) aprovechar los programas del gobierno federal en la capacitación a ejidatarios, comuneros y pequeños propietarios, para mejorar el manejo forestal y la organización para la producción y e) comenzar el proceso de transición de atribuciones en materia de gestión forestal a las entidades federativas y municipios, con la LGDFS como marco legal y de normatividad actual, y acatar la política de descentralización de funciones y recursos de la federación a los gobiernos estatales y municipales, a fin de hacerla eficaz, efectiva e integral.

Es importante destacar la oportunidad de contar con la mencionada LGDFS, toda vez que tiene concordancia con las políticas internacionales que muestran una tendencia clara hacia modelos de desarrollo acordes a los principios de sustentabilidad. 
En resumen, es clara la existencia de situaciones que están fuera de control de los actores, como el problema del tráfico de enervantes en las zonas serranas y la apertura a los mercados mundiales; sin embargo, hay amplias posibilidades de respuesta a los retos que implica el desarrollo forestal sustentable si se consideran sus capacidades, las ventajas competitivas en el estado y las oportunidades que le está brindando la federación.

Estrategia multidimensional para el desarrollo forestal sustentable en Chihuahua

Existe un avance en la visión establecida en un horizonte de cinco años o más para el desarrollo forestal de Chihuahua: integración efectiva de la cadena productiva con mejores estrategias de comercialización, rentabilidad, competitividad y empleo. A la vez, hay mayor calidad de vida y protección y conservación de los recursos, favorecidas por la participación comprometida de los actores diversos y apoyados con más colaboración interinstitucional, así como con proyectos de investigación, tecnología y programas educativos.

\section{Objetivos y acciones estratégicas comunes en el estado}

Los elementos integrados para el alcance de cada objetivo incluyen una o varias acciones estratégicas, a fin de estar en posibilidad de responder a la pregunta de cómo se alcanzará, asimismo, para cada acción se consideró responder a cuestionamientos como ¿quién llevará a cabo la acción?, ¿con qué recursos?, ¿dónde se realizará?, ¿existen necesidades de capacitación?, ¿se requiere otro tipo de apoyos? y ¿cómo se evaluarán los avances y resultados? En el cuadro 3 se presentan los objetivos y acciones estratégicas comunes, mismos que constituyen la base fundamental de la estrategia multidimensional para la búsqueda del desarrollo forestal sustentable.

Los objetivos y acciones atienden cuatro dimensiones: a) manejo sustentable de los recursos forestales (ecológica); b) creación y fortalecimiento de estructuras organizacionales, fomento a la cultura forestal y la participación activa de las comunidades en la toma de decisiones (social); c) diversificación de procesos de transformación de las materias primas forestales e incremento de la rentabilidad de las actividades (económica) y d) ejercicio de una política forestal rectora, así como la simplificación de los procesos de gestión, ante las diversas instancias relacionadas con el desarrollo (político institucional).

En la estrategia multidimensional presente se impulsa la creación y fortalecimiento de estructuras organizacionales y se reconoce la participación 
activa de las comunidades en la toma de decisiones, para consolidar el desarrollo forestal sustentable en el estado. Lo anterior está contemplado en la LGDFS, en su artículo 139, en la fracción séptima referida al impulso de la participación comunitaria para la zonificación forestal u ordenamiento ecológico como base de los programas de manejo forestal, y en la fracción novena relativa al desarrollo de la silvicultura comunitaria (Diario Oficial de la Federación, DOF 2003). De la misma manera, está muy relacionado con lo indicado en los lineamientos específicos del Programa Estratégico Forestal para México 2025 (PEF) (SEMARNAT-CONAFOR 2001), particularmente en los aspectos sociales, donde se plantea el fomento a la organización efectiva, capacitación y orientación para asegurar que las comunidades reciban los beneficios generados por el aprovechamiento de sus recursos.

\section{Temporalidad de objetivos y acciones estratégicas}

En el establecimiento de la estrategia multidimensional, se consideró la definición del horizonte de tiempo para cada uno de los objetivos y acciones estratégicas planteados, con el propósito de facilitar y orientar su ejecución futura.

Es importante aclarar que aun y cuando se consideró de cinco años o más en la definición de la visión del futuro más deseable, la naturaleza de las acciones proyectadas por los participantes ha llevado necesariamente a manejar acciones y objetivos a corto (un año), mediano (dos a cuatro) y largo plazo (cinco o más), congruentes con el establecimiento de una visión-acción como elemento clave para la búsqueda del desarrollo forestal sustentable en el tiempo.

Si se considera que la mayoría de los programas y proyectos del sector necesitan un plazo largo para concretarse, en la planeación de las actividades se requirió ajustarse a esta temporalidad, lo cual permitirá establecer un rumbo y encontrar definiciones que darán claridad y orientación precisa al trabajo. En el cuadro 4 se presenta un ejemplo de los elementos del planteamiento de temporalidad.

\section{Participación interinstitucional}

Si se considera que uno de los ejes principales de la política ambiental del Ejecutivo federal, expresado en el Plan Nacional de Desarrollo, PEF y en el Programa Nacional Forestal 2001-2006 (DOF 2002), es la participación comprometida y creativa de la sociedad, tanto para contribuir en la ejecu- 


\section{Cuadro 3}

Objetivos y acciones estratégicas comunes para el estado

\begin{tabular}{|c|c|}
\hline Objetivos & Acciones estratégicas \\
\hline $\begin{array}{l}\text { 1.Manejar } \\
\text { sustentablemente } \\
\text { los recursos forestales } \\
\text { e implementar acciones } \\
\text { para la búsqueda } \\
\text { de la certificación } \\
\text { forestal }\end{array}$ & $\begin{array}{l}\text { Elaborar y ejecutar programas de concientización y valoración de los recursos } \\
\text { forestales } \\
\text { Diseñar planes regionales para el desarrollo forestal sustentable } \\
\text { Formar comités ejidales y regionales que promuevan capacitación en materia } \\
\text { de medio ambiente } \\
\text { Asegurar el cumplimiento de las normas vigentes, } \\
\text { relacionadas con la actividad forestal } \\
\text { Establecer reglamentos internos de las comunidades, } \\
\text { para el cuidado del medio ambiente } \\
\text { Aplicar prácticas silvícolas que contribuyan a un mejor manejo } \\
\text { de los recursos forestales } \\
\text { Gestionar apoyos para la conservación de suelos, reforestación, } \\
\text { prevención y combate de incendios } \\
\text { Promover, establecer y operar unidades de manejo forestal regional (conforme } \\
\text { a la LGDFs, a través de programas y proyectos que incidan en el desarrollo forestal } \\
\text { Promover y realizar acciones tendientes a lograr la certificación forestal } \\
\text { Evaluar las condiciones ecológicas, económicas y sociales en bosques de clima } \\
\text { templado frío, mediante un sistema de criterios e indicadores para favorecer la } \\
\text { definición e implementación de estrategias orientadas al manejo forestal sustentable }\end{array}$ \\
\hline $\begin{array}{l}\text { 2.Crear y fortalecer } \\
\text { estructuras } \\
\text { organizacionales, que } \\
\text { apoyen el avance hacia el } \\
\text { desarrollo forestal } \\
\text { sustentable }\end{array}$ & $\begin{array}{l}\text { Gestionar apoyos destinados a fortalecer la organización para la producción } \\
\text { Capacitar a los productores de la región sobre la organización para la producción } \\
\text { Lograr mayor compromiso y responsabilidad de los dirigentes de cada predio } \\
\text { para mejorar la organización } \\
\text { Fortalecer procesos de autogestión para impulsar el desarrollo forestal regional } \\
\text { Crear organizaciones regionales de productores que faciliten gestiones, } \\
\text { y apoyen la adquisición de equipo y la comercialización } \\
\text { Lograr el establecimiento de microempresas comunales }\end{array}$ \\
\hline $\begin{array}{l}\text { 3.Diversificar } \\
\text { procesos de } \\
\text { industrialización, que } \\
\text { proporcionen valor } \\
\text { agregado a los productos } \\
\text { forestales, considerando el } \\
\text { aprovechamiento de los } \\
\text { subproductos }\end{array}$ & $\begin{array}{l}\text { Organizar a los productores para la adquisición de equipo para industrializar } \\
\text { la madera y darle valor agregado } \\
\text { Capacitar a los productores forestales sobre diversos procesos de industrialización } \\
\text { de las materias primas } \\
\text { Gestionar créditos para equipamiento de industrias como aserraderos, estufas, } \\
\text { impregnadoras y tableteras, entre otros } \\
\text { Elaborar un diagnóstico para determinar la utilización de residuos } \\
\text { de los productos maderables } \\
\text { Implementar la reconversión industrial }\end{array}$ \\
\hline $\begin{array}{l}\text { 4.Fomentar la } \\
\text { cultura forestal, para } \\
\text { impulsar el desarrollo } \\
\text { forestal sustentable }\end{array}$ & $\begin{array}{l}\text { Integrar el tema de cultura forestal y de protección al medio ambiente en los } \\
\text { programas de educación } \\
\text { Proponer ante la Secretaría de Educación Pública (SEP) la inclusión en las currículas } \\
\text { contenidos y materias relacionados con los recursos forestales }\end{array}$ \\
\hline $\begin{array}{l}\text { 5. Generar investigación y } \\
\text { tecnología para el manejo } \\
\text { integral de los recursos, } \\
\text { y aumentar la producción } \\
\text { y productividad del sector } \\
\text { forestal }\end{array}$ & $\begin{array}{l}\text { Elaborar un diagnóstico de las necesidades de investigación } \\
\text { Establecer programas de investigación en áreas estratégicas prioritarias } \\
\text { Establecer procesos de seguimiento, aplicación y divulgación de programas } \\
\text { de investigación }\end{array}$ \\
\hline $\begin{array}{l}\text { 6.Garantizar la } \\
\text { participación activa de las } \\
\text { comunidades, incluso } \\
\text { las indígenas en la toma } \\
\text { de decisiones de las } \\
\text { actividades } \\
\text { consideradas para el } \\
\text { desarrollo forestal } \\
\text { sustentable }\end{array}$ & $\begin{array}{l}\text { Capacitación que favorezca la aplicación de procesos participativos } \\
\text { como base de autogestión } \\
\text { Crear reglamentos internos de ejidos y comunidades }\end{array}$ \\
\hline $\begin{array}{l}\text { 7. Incrementar la } \\
\text { rentabilidad de las } \\
\text { actividades forestales }\end{array}$ & $\begin{array}{l}\text { Mejorar la elaboración y operación de los programas de manejo forestal } \\
\text { Incrementar la productividad en las labores de abastecimiento } \\
\text { Diversificar y modernizar la industria }\end{array}$ \\
\hline
\end{tabular}




\section{Continuación del cuadro 3}

\begin{tabular}{|c|c|}
\hline Objetivos & Acciones estratégicas \\
\hline $\begin{array}{l}\text { 8. Optimizar la } \\
\text { comercialización } \\
\text { de los productos forestales }\end{array}$ & $\begin{array}{l}\text { Determinar las necesidades del consumidor sobre productos } \\
\text { Identificar los nichos de mercado } \\
\text { Definir los canales de comercialización }\end{array}$ \\
\hline $\begin{array}{l}\text { 9. Simplificar los procesos } \\
\text { de gestión ante diversas } \\
\text { instancias relacionadas } \\
\text { con el desarrollo forestal }\end{array}$ & $\begin{array}{l}\text { Unificar criterios entre instancias que faciliten los procesos de gestión } \\
\text { Propiciar la generación de normas mexicanas que contribuyan a facilitar } \\
\text { la toma de decisiones en los diversos trámites }\end{array}$ \\
\hline $\begin{array}{l}\text { 10. Capacitar a } \\
\text { productores sobre el } \\
\text { manejo de los recursos } \\
\text { naturales, educación y } \\
\text { salud, entre otros }\end{array}$ & $\begin{array}{l}\text { Aplicar encuestas a productores, personal técnico e iniciativa privada, sobre sus } \\
\text { necesidades de capacitación para la elaboración de programas y cursos teóricos } \\
\text { y prácticos } \\
\text { Constituir legalmente centros regionales de capacitación } \\
\text { Gestionar apoyos gubernamentales en los tres órdenes para la creación de centros } \\
\text { regionales de capacitación } \\
\text { Coordinar la construcción o gestión de infraestructura para operar los centros } \\
\text { regionales de capacitación } \\
\text { Adquirir el terreno o local o gestionar infraestructura existente para operar los } \\
\text { centros regionales de capacitación } \\
\text { Contratar personal técnico docente para laborar en los centros regionales de } \\
\text { capacitación } \\
\text { Poner en marcha el programa de capacitación, mediante cursos impartidos a } \\
\text { productores forestales del estado } \\
\text { Impartir talleres, conferencias y cursos de capacitación }\end{array}$ \\
\hline $\begin{array}{l}\text { 11. Elevar el nivel de } \\
\text { educación y salud de los } \\
\text { habitantes de las zonas } \\
\text { forestales }\end{array}$ & $\begin{array}{l}\text { Promover y fomentar programas culturales y de salud } \\
\text { Crear escuelas técnicas y superiores }\end{array}$ \\
\hline $\begin{array}{l}\text { 12. Crear fuentes de } \\
\text { empleo alternas a la } \\
\text { actividad forestal, que } \\
\text { contribuyan a mejorar la } \\
\text { calidad de vida de los } \\
\text { pobladores. }\end{array}$ & $\begin{array}{l}\text { Impartir talleres participativos para definir propuestas y necesidades de fuentes } \\
\text { de empleo, y buscar usos alternativos de los recursos } \\
\text { Gestionar, elaborar e implementar proyectos productivos para la creación } \\
\text { de fuentes de empleo con diferentes actividades económicas } \\
\text { Adquirir equipo para el desarrollo de la industria forestal financiada } \\
\text { por el gobierno y que genere empleos } \\
\text { Establecer centros de producción de los recursos asociados con apoyos del gobierno } \\
\text { Crear sociedades con inversionistas para producir artesanías }\end{array}$ \\
\hline $\begin{array}{l}\text { 13. Integrar la cadena } \\
\text { productiva forestal para } \\
\text { mejorar la } \\
\text { competitividad }\end{array}$ & $\begin{array}{l}\text { Eficientar los procesos de extracción de materia prima y su transformación } \\
\text { Gestionar con los tres órganos de gobierno la rehabilitación de la red caminera } \\
\text { en las regiones forestales } \\
\text { Organizar a los productores para rehabilitación de caminos } \\
\text { Capacitar a productores en extracción industria y comercio de productos } \\
\text { Realizar estudios de mercados para los productos } \\
\text { Gestionar recursos financieros para producción forestal y adquisición de equipo } \\
\text { ante instituciones como Fideicomisos Instituidos en Relación con la Agricultura } \\
\text { (FIRA), Banco Nacional de Comercio Exterior (BANCOMEXT) y FIDEICOMISO, entre otras }\end{array}$ \\
\hline $\begin{array}{l}\text { 14. Incrementar, simplificar } \\
\text { y gestionar subsidios } \\
\text { directos para proyectos } \\
\text { productivos que impulsen } \\
\text { el desarrollo de la región }\end{array}$ & $\begin{array}{l}\text { Solicitar asesoría a diferentes instancias de gobierno para acceder a los programas } \\
\text { vigentes tales como: PRODEFOR, RODEPLAN, entre otros } \\
\text { Gestionar apoyos que existen en la CONAFOR, Gobierno del Estado, asociación de } \\
\text { productores forestales y Fideicomiso Chihuahua Forestal, para plantaciones } \\
\text { y preaclareos, entre otros } \\
\text { Gestionar ante instituciones el incremento y simplificación de apoyos } \\
\text { para impulsar el desarrollo de la región }\end{array}$ \\
\hline $\begin{array}{l}\text { 15. Crear fondos de } \\
\text { ahorro en ejidos y } \\
\text { comunidades forestales } \\
\text { para cuidar el bosque } \\
\text { y su infraestructura }\end{array}$ & $\begin{array}{l}\text { Hacer concientes a los productores sobre las ventajas y necesidad } \\
\text { de los fondos de ahorro } \\
\text { Crear un fondo de ahorro en cada uno de los ejidos y comunidades } \\
\text { de la región forestal del estado } \\
\text { Elaborar reglamentos de operación para los fondos de ahorro }\end{array}$ \\
\hline $\begin{array}{l}\text { 16. Fomentar la } \\
\text { participación de gestores } \\
\text { para trámites diversos que } \\
\text { favorezcan el desarrollo } \\
\text { de la región }\end{array}$ & $\begin{array}{l}\text { Identificar y elegir al gestor, a través de los mecanismos establecidos } \\
\text { por las comunidades y ejidos } \\
\text { Formar un fondo para acciones de gestoría para el desarrollo de la región }\end{array}$ \\
\hline
\end{tabular}




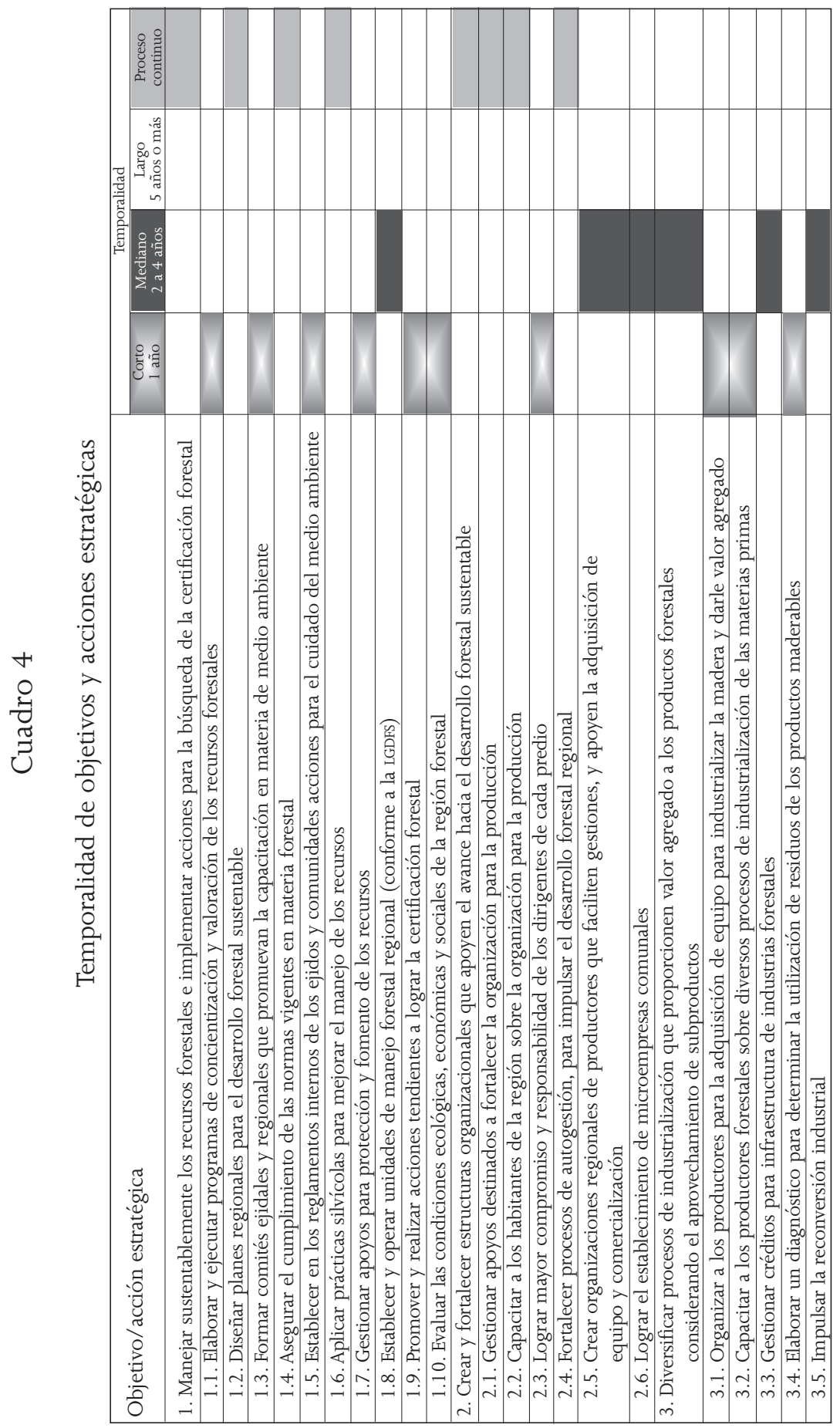




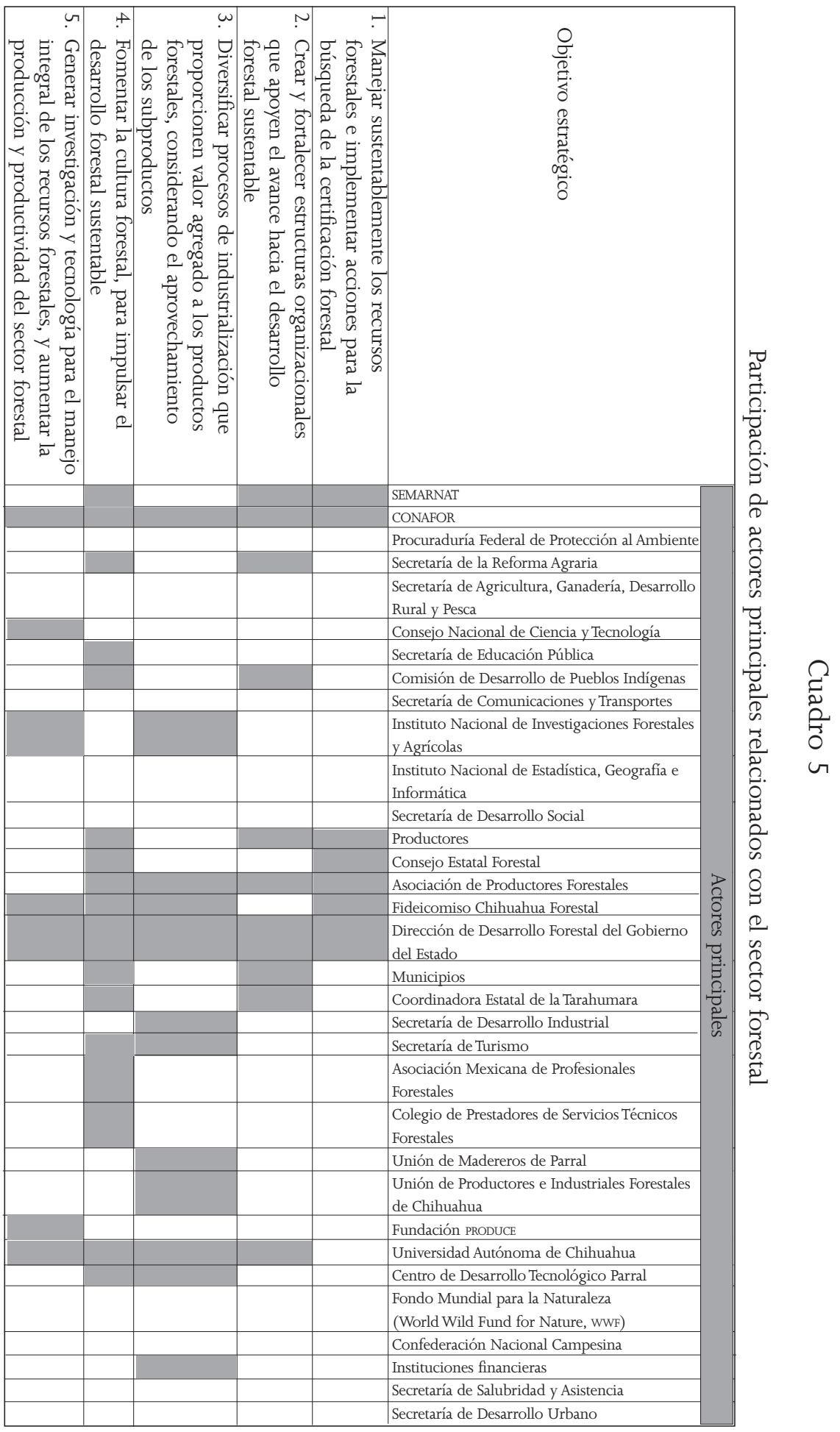


ción de diversos programas, como para dar seguimiento y evaluar el desempeño de las autoridades, en esta estrategia se planteó, además de la definición de objetivos y acciones y su temporalidad, definir la posible intervención de instituciones relacionadas con el sector forestal, para el establecimiento futuro de las acciones propuestas. Así, esta definición permitirá visualizar las actividades que requiere un proceso de coordinación de esfuerzos y recursos económicos que coadyuven a su implantación.

En el proceso de planeación del desarrollo del sector forestal es importante abordar el tema de la coordinación institucional, en virtud de que en Chihuahua existe una gran diversidad de instancias de los tres órdenes de gobierno que participan e inciden en mayor o menor medida en el proceso. También existen organizaciones no gubernamentales que intervienen activamente en algunos proyectos relacionados con el manejo de recursos naturales y ambientales, razón por la cual se consideró necesaria su colaboración en la integración de la estrategia multidimensional. En el cuadro 5 se muestra un ejemplo del contenido del planteamiento de la participación interinstitucional.

\section{Mecanismos para la implementación y evaluación de la estrategia multidimensional}

Con la finalidad de que se realice la puesta en marcha y evaluación de la estrategia de la mejor manera y se beneficie a los actores involucrados, a continuación se plantean mecanismos específicos, afines a lo planteado en los instrumentos existentes de política forestal nacional y estatal, como son el PEF, la LGDFS, el Programa Proárbol de la Comisión Nacional Forestal y el Programa de Desarrollo Forestal Sustentable de Chihuahua, entre otros:

- El CEFECH decidió integrar esta estrategia multidimensional con el Programa de Desarrollo Forestal Sustentable del Estado de Chihuahua, elaborado por el gobierno estatal en 2004. Para ello, definió y creó un comité de seguimiento y evaluación, para establecer los mecanismos de ejecución, responsabilidad, coordinación, cooperación y financiamiento del programa, que permita su establecimiento en el corto, mediano y largo plazo, para alcanzar los objetivos propuestos.

- Para implantar la estrategia multidimensional, se propone la elaboración de programas y proyectos regionales que impulsen el desarrollo forestal sustentable, considerando los objetivos y acciones estratégicas identifica- 
dos, así como los registrados por los ejidatarios, comuneros y pequeños propietarios en los planes particulares de cada región.

- Para el diseño y elaboración de los programas y proyectos se recomienda considerar la regionalización establecida por la CONAFOR Región vi "Río Bravo”, a través de las unidades de manejo forestal de acuerdo a lo establecido en la LGDFS (DOF 2003).

- Para el diseño, puesta en marcha y evaluación de los programas y proyectos regionales se propone considerar la MCB como una metodología de planeación estratégica participativa, la cual brinda una alternativa importante para idear e implantar un proceso sistematizado, que puede apoyar el desarrollo regional forestal con una visión de sustentabilidad.

- Según los proyectos y programas regionales generados, se sugiere precisar las posibles instancias para su financiamiento y ejecución, que pueden ser gubernamentales y no gubernamentales, nacionales e internacionales.

- Es recomendable establecer un sistema jerárquico de criterios e indicadores de sustentabilidad, con variables clave medibles, que indiquen la situación actual de los aspectos ecológicos, sociales y económicos de la región del bosque de clima templado frío del estado. Dicho sistema debe de constituir una base fundamental para efectuar el seguimiento de la dinámica y avance hacia un desarrollo forestal sustentable, a través de la realización de evaluaciones estratégicas periódicas.

- Según ocurran los cambios continuos y dinámicos del entorno y del mismo sector forestal, se propone realizar ajustes periódicos, cada dos años, a la estrategia multidimensional, acorde con su naturaleza, tanto en el sector forestal como en su ambiente externo. Lo anterior permitirá adecuar los objetivos y acciones estratégicas en el tiempo y espacio.

Recibido en mayo de 2007 Revisado en septiembre de 2007

\section{Bibliografía}

Bray Barton, David, Leticia Merino-Pérez, Patricia Negreros-Castillo, Gerardo Segura-Warnholtz, Juan Manuel Torres-Rojo y Henricus F. M. Vester. 2003. Mexico's Community-Managed Forests as a Global Model for Sustainable Landscapes. Conservation Biology 17 (3): 672-677. 
Consejo Técnico Consultivo Forestal de Chihuahua. 1996. Programa Estatal de Desarrollo Sustentable Forestal. Resumen ejecutivo presentado al consejo en sesión del 15 de abril, Chihuahua.

Diario Oficial de la Federación. 2003. Ley General de Desarrollo Forestal Sustentable. 25 de febrero.

2002. Programa Nacional Forestal 2001-2006. 27 de septiembre.

Emery, Merrelyn y Ronald E. Purser. 1996. The Search Conference:A Powerful Method for Planning Organizational Change and Community Action. San Francisco: JosseyBass Publisher.

Fideicomiso Chihuahua Forestal. 2003. Ubicación de asociaciones de productores forestales en el estado de Chihuahua. Chihauhua.

Guerrero, María Teresa, Cyrus Reed y Brandon Vegter. 2000a. La industria forestal y los recursos naturales en la Sierra Madre de Chihuahua: impactos sociales, económicos y ecológicos. Chihuahua: Comisión de Solidaridad y Defensa de los Derechos Humanos, A.C. y Texas Center for Policy Studies.

Guerrero, María Teresa, Francisco Villa, Mary Kelly, Cyrus Reed y Brandon Vegter. 2000b. La industria forestal en el estado de Chihuahua: impactos económicos, ecológicos y sociales después del tLCAN. Chihuahua: Comisión de Solidaridad y Defensa de los Derechos Humanos, A.C. y Texas Center for Policy Studies.

Luján Álvarez, Concepción, Jesús Miguel Olivas García, Salvador Balderrama Castañeda, Juan Manuel Chacón Sotelo, Juan Manuel Baca Venegas, Eduardo Arrieta Solís e Hilda Guadalupe González H. 2006. Desarrollo forestal sustentable en la cuenca del río Papigochi, estado de Chihuahua. Chihuahua: Universidad Autónoma de Chihuahua.

Luján Álvarez, Concepción. 2001. Protocolo para el diseño del plan estratégico participativo para el desarrollo forestal sustentable en Chihuahua. Delicias: Universidad Autónoma de Chihuahua.

Luján Álvarez, Concepción, Joel Diemer y Lois Stanford. 2000. Desarrollo de comunidades forestales en Chihuahua, México. Revista Madera y Bosques 6 (2): 29-39. 
Secretaría de Agricultura y Recursos Hidráulicos. 1994. Inventario forestal periódico del estado de Chihuahua. México: Subsecretaría Forestal y de la Fauna Silvestre.

Secretaría de Medio Ambiente y Recursos Naturales-Comisión Nacional Forestal. 2001. Programa Estratégico Forestal para México 2025. México: SEMARNATCONAFOR.

Vanhanen, Heidi, Gerardo Mery, Sebastiao Kengen, Jussi Uusivuori, Susanna Laaksonen-Craig y Anne Toppinen. 2005. Responding to Increasing Social and Economic Demands on Forests. En Forests in the Global BalanceChanging Paradigms, editado por G. Mery, R. Alfaro, M. Kanninen y M. Lobovikov, 21-38. Helsinki: Unión Internacional de Organizaciones de Investigación Forestal (IUFRO, por sus siglas en inglés). 
